\title{
Adequate Theory of Oscillator: A Prelude to Verification of Classical Mechanics Part 4
}

\author{
Zdzisław Pluta, Tadeusz Hryniewicz* \\ Faculty of Mechanical Engineering , Koszalin University of Technology, \\ Racławicka 15-17, 75-620 Koszalin, Poland \\ *E-mail address: Tadeusz.Hryniewicz@tu.koszalin.pl
}

"The essence of scientific discovery relies on the fact that one looks at the same what everyone sees and notices what nobody has seen."

L. Pauling

\begin{abstract}
In the paper, the adequate theory of oscillator is presented, being a sort of prelude to verification of the classical theory of mechanics. The developed theory is based on a properly understood notion of energy, quantum value changes of its determined measures (potentials), as well as of such changes types of sites of full energetic states which presents the essence of the true principle of the energy conservation. In the first part of the paper the principle of energy conservation was considered. Then the energetic aspects of the oscillator motion, with an exemplary real system motion was presented. The third part was a development of kinetics of a body in the harmonic motion and verification of the adequate theory of the oscillator. At the end, this Part 4 is devoted to the determination of the gravity acceleration by means of the mathematical pendulum to confirm the previously presented findings.
\end{abstract}

\section{Keywords:}

Oscillator; Energy; Principle of energy conservation; Space-time; Potential field; Gravitation

\section{INTRODUCTION}

First two parts of the paper served to introduce readers into the problems of verification of the classical mechanics [1,2]. General characteristics of the classical mechanics were presented based on the references. Exemplification of artifacts of the classical theory of oscillator was developed. Part 2 of the paper was to consider the energetic aspects of oscillator motion, by description of an exemplary real system motion in reference to the existent differential equation of the oscillator motion. Part 3 covered the kinetics of a body 
under the harmonic motion and verification of the adequate theory of oscillator [3]. This Part 4 of the paper is to show the results of another method for determination of the gravity acceleration, compare with the first one, and sum up the new achievements in the mechanics.

\section{DETERMINATION OF THE GRAVITY ACCELERATION BY MEANS OF MATHEMATICAL PENDULUM}

The gravity acceleration has been decided to be determined one more time, with other method, to obtain further confirmation of the adequacy for the determined value of that, surely an essential physical magnitude. A very popular method has been chosen, used primarily for this task. This is the method of mathematical pendulum, as a sort of the string angular oscillator. Surely one cannot assume this existent theory of pendulum because it arose on the classical ground and possesses a determined fault/defect, in the way as it is in reference to the existent classical theory of the spring harmonic oscillator. Here also the energetic quantum approach to the described reality has been used. Anyway one cannot proceed in other way because of the characteristic feature of the nature which is just quantum [8-11]. Here only that part of the theory which may be directly used to determine the gravity acceleration is given. This has been derived basing on the scheme (Fig. 15).

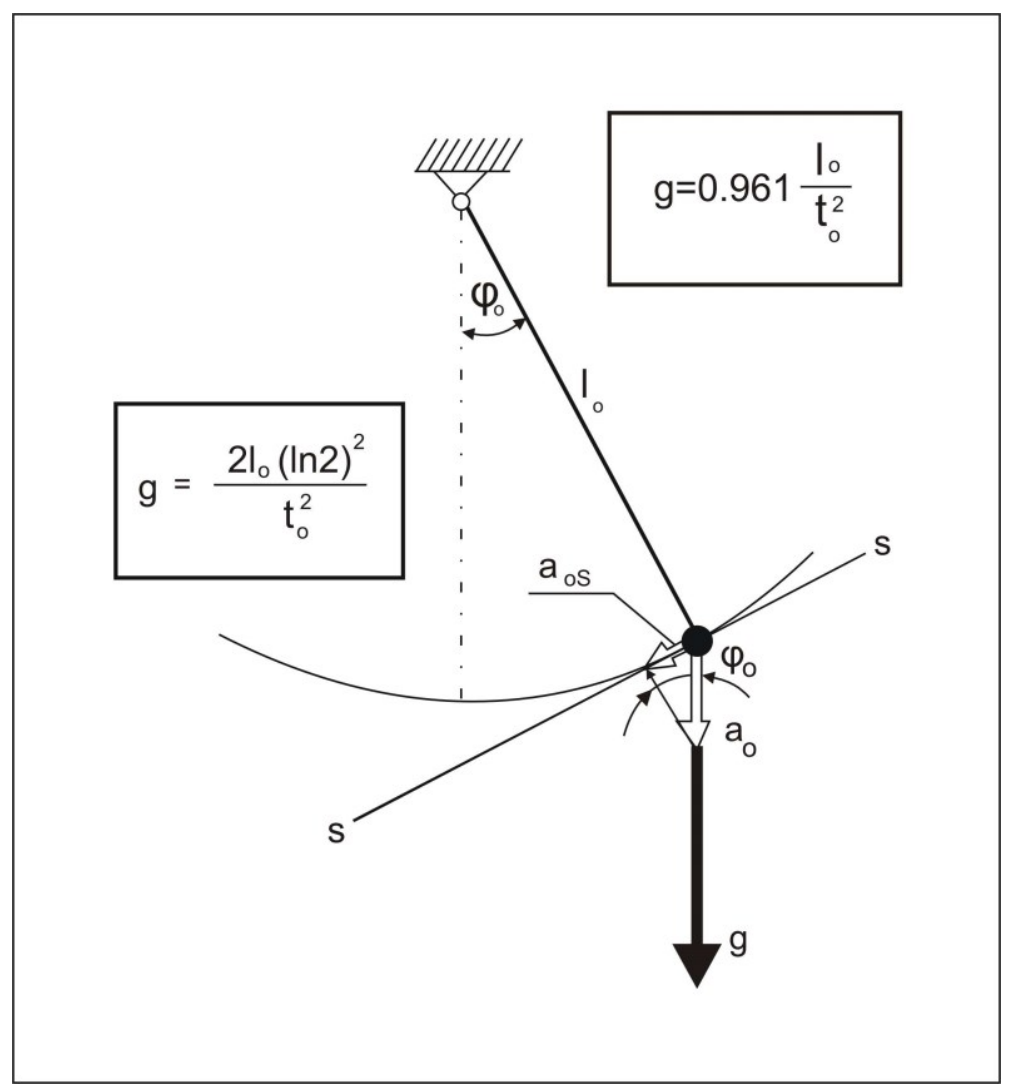

Fig. 15. Scheme of a part of mathematical pendulum theory for derivation the formula on gravity acceleration 
A point material body is in the angular unstable position where its inertia is distinctly emphasized/set off. Only the tangent component of the initial inertia force $a_{o s}$ has been marked. The body has its fixation/fastening on the grappled, in a determined point, the string of length $l_{o}$. It has been deflected at the angle $\varphi_{o}$ from the vertical position which corresponds with a stable energetic state of the system.

After the body release from the unstable position it will be permeating the space-time with the accelerated motion, determined by the mentioned angle. The length of the angular way/trajectory is described by the following relationship:

$$
\varphi=\varphi_{0}\left(e^{\frac{t}{T}}-1\right)
$$

The angular acceleration $\varepsilon$, being the second derivative of the former magnitude, possesses the following analytical record:

$$
\varepsilon=\ddot{\varphi}=\frac{d^{2} \varphi}{d t^{2}}=\frac{\varphi_{0}}{T^{2}} e^{\frac{t}{T}}=\varepsilon_{0} e^{\frac{t}{T}}
$$

where $\varepsilon_{o}$ denotes an initial angular acceleration; all other parameters are known yet [1-3].

After the transition of the body through the space-time, for $t_{o}=T \ln 2$, there will be the following relation between the extremes of the accelerations:

$$
\varepsilon^{*}=\varepsilon_{0} e^{\frac{T \ln 2}{T}}=2 \varepsilon_{0}=\frac{2 \varphi_{0}}{T^{2}}
$$

Now one should get to the linear direction $s-s$, tangent to the trajectory of the body motion. That means the angular acceleration should be bound with the linear acceleration. Thus one obtains:

$$
a_{0}=\varepsilon_{0} l_{0}=\frac{g}{2} \sin \varphi_{0}
$$

and

$$
\varepsilon_{0}=\frac{g}{2 l_{0}} \sin \varphi_{0}
$$


Now by inserting (41) to the two former records of formula (39), the following relationship is obtained:

$$
2 \frac{g}{2 l_{0}} \sin \varphi_{0}=\frac{2 \varphi_{0}}{T^{2}}
$$

After the accomplishment of appropriate simple operations, and also taking into account the equality for small angles, i.e. $\varphi_{o}=\sin \varphi_{o}$, one obtains:

$$
\frac{g}{l_{0}}=\frac{2}{T^{2}}
$$

and finally

$$
g=\frac{2 l_{0}}{T^{2}}
$$

and after taking into account the relation $t_{o}=T \ln 2$, one obtains the formula on the gravity acceleration, which can be used directly in practice, then:

$$
g=\frac{2 l_{0}(\ln 2)^{2}}{t_{o}^{2}}
$$

or

$$
g=0.961 \frac{l_{0}}{t_{0}^{2}}
$$

By substituting the experimental data: $l_{o}=1 \mathrm{~m}, t_{o}=0.5 \mathrm{~s} ; l_{o}=0.5 \mathrm{~m}, t_{o}=0.35 \mathrm{~s} ; l_{o}=$ $0.25 \mathrm{~m}, t_{o}=0.25 \mathrm{~s} ; l_{o}=0.125 \mathrm{~m}, t_{o}=0.18 \mathrm{~s} ; l_{o}=0.111 \mathrm{~m}, t_{o}=0.175 \mathrm{~s}$; to the formula (46) as a result one obtains the following values of the acceleration: $g_{1}=3.844 \mathrm{~m} \cdot \mathrm{s}^{-2}, g_{2}=3.922 \mathrm{~m} \cdot \mathrm{s}$ ${ }^{2}, g_{3}=3.844 \mathrm{~m} \cdot \mathrm{s}^{-2}, g_{4}=3.71 \mathrm{~m} \cdot \mathrm{s}^{-2}$, and $g_{5}=3.918 \mathrm{~m} \cdot \mathrm{s}^{-2}$. Finally, as the average of these results, the analogous one as before [3] the result has been obtained, namely $g=3.85 \mathrm{~m} \cdot \mathrm{s}^{-2}$.

Both considered methods have been set up and confronted (Fig. 16), by presenting proper, corresponding to them, the formulae on the gravity acceleration, and the value of that physical magnitude has been placed in the center of the Fig. 16. 


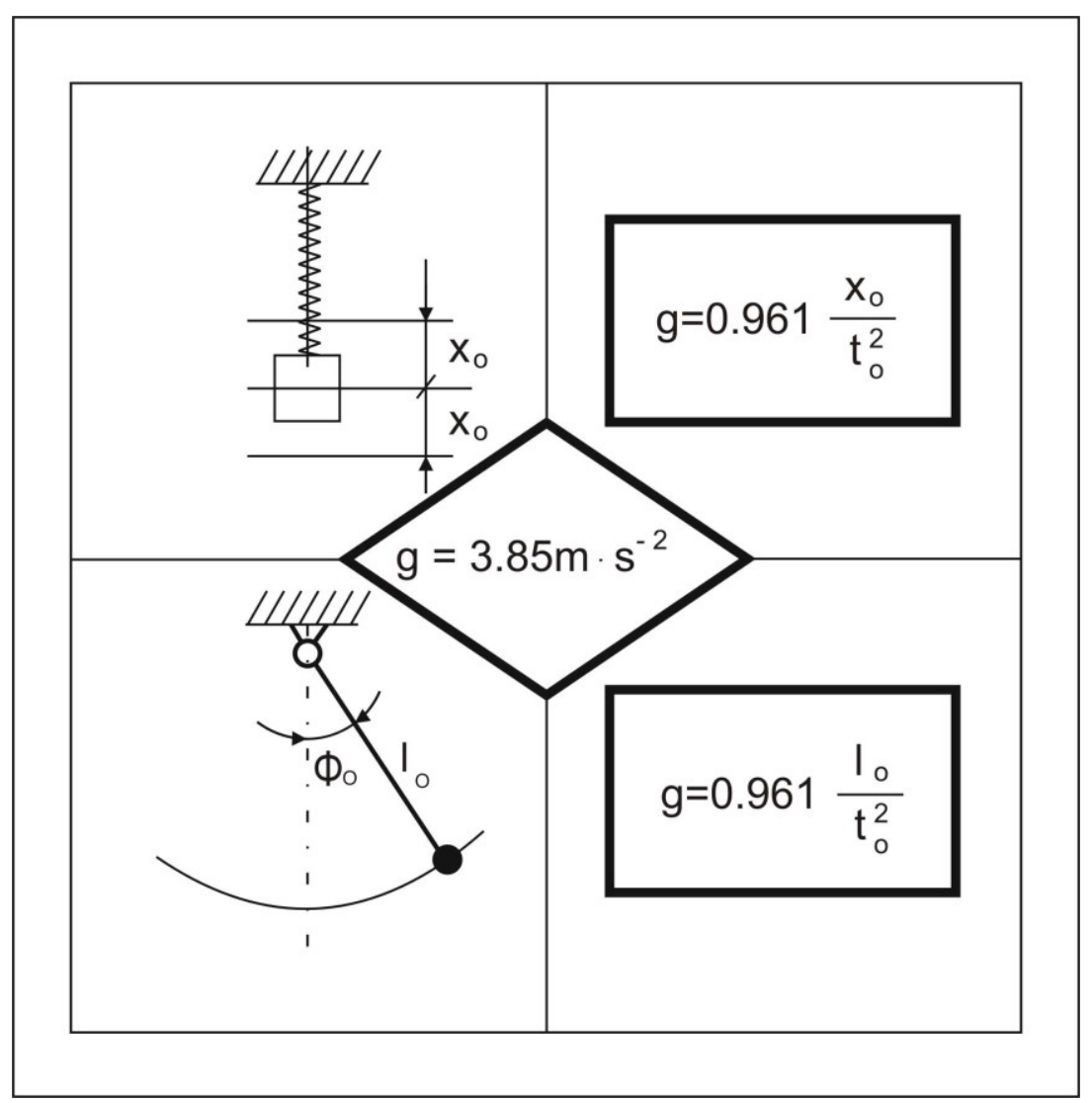

Fig. 16. Schemes of the harmonic body motion and corresponding formulae on the gravity acceleration

\section{CONCLUSION}

In conclusion it is worth stating that the presented papers (Parts 1 though 4) indicate directly on the quantum character of the considered phenomena, as may be judged on the basis of these considerations; in fact one may assume all the phenomena are taking place in the nature [8-11].

Therefore there is no place for so called a classical mechanics which, together with the quantum mechanics, still forms a kind of "bimechanics" (by analogy to e.g. bimetal). Until this time both the mechanics function one by another, presenting different, quite separate look and approach to the reality. The classical mechanics, as such, assumes the continuity of energy variation, whereas the second one states a jump, quantum changes of this magnitude. Meanwhile the energy changes only by the quantum. The classical approach should be concerned rather on the work only which has been identified with energy. There should be one mechanics only, without additional qualifications or designations which divide them quite non-reasonably.

As given it the title, this is only a prelude to the verification of classical mechanics. The existent classical knowledge of reality is bristled up with the elements of mathematic creation to such a degree that it lost its character of an adequate knowledge. Thus the classical mechanics should be verified by detachment/irrelevance from the main still developed 
speculative approach/direction, forming a loop of verification crossing through the source where the truth of reality is situated.

In the light of this investigation, one should refer to the expressed regret in the work [7]: "Nobody until now has formulated an appropriate quantum theory of the gravity, whereas a graviton, or a quantum of gravitation, remains the notion of poor appreciation and is rather elusive. An attempt to unify the gravitation with other natural reactions seems to be futile/vain as one can hardly obtain a homogeneous/uniform theory of which one part is quantum but not the other".

And this is the state-of-art referred to mechanics, or in fact "bimechanics" as earlier noticed. No strange that a quantum of the gravitation is incomprehensible, poorly perceptible if also the energy is still so enigmatically explained and identified with work. Nothing strange, because the Newton's law refers to the imaginative reality [12], and the force of gravity is projected or completely eliminated. That last operation has been used in introduction of a classical equation of the oscillator motion. Quantum theory of gravitation? Why talking about a quantum theory of this phenomenon by tolerating at the same time a non-quantum theory? Gravitation is in fact, quantum and the theory of touching this phenomenon should be one and adequate.

At the end it is worth admitting that one cannot consider the gravitation in separation from inertia which unfortunately is recognized as a fictitious thing by introducing its measure as the fictitious inertia force (d'Alembert principle). Therefore one cannot say just about a graviton, or rather gravitational potential, but also of inertion or inertive potential, as well as of other potentials which characterize energetic states of particular elements of the whole considered system of material bodies. All of these things are to be considered and discussed in further Authors' works, in the near future.

\section{References}

[1] Zdzisław Pluta, Tadeusz Hryniewicz, International Letters of Chemistry, Physics and Astronomy 2 (2012) 28-34.

[2] Zdzisław Pluta, Tadeusz Hryniewicz, International Letters of Chemistry, Physics and Astronomy 3 (2012) 1-10.

[3] Zdzisław Pluta, Tadeusz Hryniewicz, International Letters of Chemistry, Physics and Astronomy 3 (2012) 11-23.

[4] Zdzisław Pluta and Tadeusz Hryniewicz, Intern. J. Adv. Mater. Technol. 62(5) (2012) 529-542; DOI: 10.1007/s00170-011-3813-5.

[5] L.D. Landau, J.M. Lifszyc, Theoretical Physics. Mechanics (transl. from Russian). $4^{\text {th }}$ edition, PWN SA, Warszawa 2006.

[6] K. Chyla, Physics (in Polish). Edited by DEBIT, Bielsko Biała 1996.

[7] J. Magueijo, Faster than light (transl. from English). Edited by Amber, Warsaw 2003.

[8] Zdzisław Pluta, Energetyka 7 (2005) 496-501, (in Polish),

[9] Zdzisław Pluta, LAB 2 (2004) 50-52, (in Polish), 
[10] Zdzisław Pluta, LAB 6 (2004) 42-46, (in Polish),

[11] Zdzisław Pluta, LAB 3 (2004) 40-43, (in Polish),

[12] Zdzisław Pluta, LAB 2 (2005) 45-48, (in Polish). 